\title{
Le soutien à la diffusion des innovations radicales pour le transport de marchandises : les bonnes pratiques issues de la comparaison franco-allemande
}

\author{
Promotion of the diffusion of radical innovations in goods transportation \\ system: best practices from the German-French comparison
}

\author{
Stephan Müller ${ }^{1}$, Corinne Blanquart ${ }^{2}$ \\ ${ }^{1}$ DLR-IVF, Germany, Stephan.mueller@dlr.de \\ ${ }^{2}$ Université Paris-Est, IFSTTAR, SPLOTT, France, corinne.blanquart@ifsttar.fr
}

\begin{abstract}
RÉSUMÉ. L'article questionne les conditions d'émergence et de diffusion des innovations radicales en matière de transport de marchandises, au regard de la permanence des nuisances environnementales associées à cette activité. II montre la nécessité de considérer l'innovation de rupture comme une innovation sociale, amenant à re-considérer l'organisation des relations entre les acteurs du système de transport, qu'il convient dès lors d'associer au processus d'innovation. De même, le renouvellement du rôle de l'Etat, au travers de structures pertinentes d'interface entre science et industrie, y est réaffirmé. L'article illustre dès lors les spécificités nationales entre la France et l'Allemagne à la fois dans les partenariats propres au processus d'innovation, que dans le rôle de l'Etat. ABSTRACT. The topic of this article concerns the conditions for the emergence and dissemination of radical innovations in freight transport, with regard to environmental externalities. It shows the need to consider radical innovation as a social innovation, leading to reconsidering the organization of the relationships between the actors of the transport system, who should therefore be associated to the innovation process. Similarly, the renewal of the role of the state, through relevant interfaces between science and industry, is reaffirmed. The article thus illustrates the national specificities between France and Germany both in the partnerships in the innovation process and in the role of the state.

MOTS-CLÉS. Innovation radicale, transport de marchandises, innovation sociale, systèmes nationaux d'innovation.

KEYWORDS. Radical innovation, freight transport, social innovation, national innovation systems.
\end{abstract}

\section{Introduction}

En décalage par rapport à son image, le transport est l'un des secteurs les plus innovants en Europe. L'Europe et les Etats ont ainsi massivement investi dans l'innovation en matière de transport, en raison du poids économique de ce secteur en termes d'emploi, mais aussi au regard de sa contribution au PIB ou de son rôle attendu sur la cohésion économique et sociale. De plus, les entreprises européennes de ce secteur sont des acteurs majeurs sur la scène mondiale. Enfin, les préoccupations environnementales et énergétiques appellent des évolutions du système de transport qui reposent sur l'innovation. Toutefois, comme le souligne J.Theys (2005), " les informations dont on dispose sur leur coût, leur impact, leur marché potentiel, leur difficulté de mise en oeuvre... sont - sauf exception - très fragmentaires ».

L'innovation est également un levier traditionnel des politiques de transport durable, à toutes les échelles, y compris européenne. Toutefois, malgré une historicité de mise en œuvre des politiques d'innovation, les problèmes environnementaux associés au transport demeurent. Des travaux mettent alors en avant la nécessité d'innovations plus radicales, sans pour autant remettre en cause ce levier d'intervention.

L'ambition de l'article est alors d'examiner à travers un certain nombre de projets quelles sont les conditions d'émergence de ces innovations plus radicales.

L'analyse comparative entre la France et l'Allemagne s'avère intéressante dans la mesure où les 2 pays mènent des politiques industrielles différentes et où la structure du système de transport de marchandises est elle aussi différente, à la fois dans les parts modales et dans les jeux d'acteurs. 
Il s'agit par conséquent d'envisager les déterminants des innovations radicales dans les deux pays, afin d'éclairer les outils capables d'accompagner la diffusion de ces innovations, en favorisant l'évolution dans l'organisation du système d'acteurs qui structure le système de transport de marchandises.

Nous présenterons dans un premier temps les spécificités de l'innovation en transport, notamment de marchandises, et les cadres d'analyse que nous mobiliserons.

Nous détaillerons ensuite la méthodologie utilisée et les projets qui ont été analysés, avant de présenter les principaux résultats, nous permettant de formuler des recommandations pour promouvoir l'innovation en transport de marchandises.

\section{L'innovation radicale en transport de marchandises : enjeux et cadres d'analyse}

L'émergence et la diffusion des innovations sont envisagées comme des vecteurs majeurs de croissance et de productivité. En matière de transport, les enjeux de développement durable en ont fait un levier important des politiques de transport durable: " l'innovation technologique est une opportunité à saisir pour intégrer les modes de transport, optimiser leur performance, les rendre plus sûrs et contribuer à rendre le système européen de transports compatible avec le développement durable des transports » (annexe 4 du livre blanc de 2001).

Toutefois, parmi les éléments identifiés par Kingdon (1995) pour expliquer la survenue de changements plus radicaux, est mentionnée l'apparition d'anomalies que le paradigme en vigueur ne permet pas de prendre en compte et qui engendrent une pression pour leur résolution. La permanence des nuisances environnementales du transport peut constituer une telle anomalie. La Commission Européenne continue en effet de faire le constat que dans le domaine de l'environnement, des progrès supplémentaires sont nécessaires. "Dans l'UE, si on compare par rapport aux niveaux de 1990, le secteur des transports est celui où le taux de croissance des GES est le plus élevé. Si l'on applique cette analyse à des évolutions passées dans le domaine des transports, on constate que l'activité de ce secteur a considérablement augmenté, alors que les progrès dans le sens d'une réduction de sa consommation d'énergie des émissions de GES ont été insuffisants » (CCE, 2009).

Le développement durable semble alors un élément suffisamment perturbateur, pour être assimilé aux anomalies que Kingdon (1995) estime susceptibles de générer des changements radicaux.

Ce sont en effet "les ruptures technologiques radicales qui autorisent véritablement une transformation du système productif, une modification des modes de consommation et des modes de vie en amorçant une nouvelle trajectoire technologique qui finit par coïncider avec une trajectoire de développement durable » (Faucheux, Nicolaï, 1998). Les innovations radicales en transport et en transport de marchandises présentent toutefois un certain nombre de spécificités, qu'il convient d'éclairer.

La question consiste en effet à déterminer l'origine de la rupture propre à l'innovation. On peut toutefois distinguer plusieurs types d'innovations :

- Les innovations incrémentales qui sont des améliorations mineures des produits et des processus ;

- Les innovations radicales associées à une modification de grande ampleur de l'ensemble des processus, et qui présentent un caractère irréversible ;

-Les révolutions technologiques qui sont le résultat de plusieurs innovations radicales et qui affectent l'économie dans son ensemble et pas une seule entreprise.

La frontière est ténue entre innovations incrémentales et radicales, un ensemble d'innovations incrémentales pouvant conduire à une rupture. Toutefois, nous nous concentrerons ici sur les innovations radicales au regard de la permanence des problèmes environnementaux associés au transport, et ce malgré les nombreux programmes de soutien à l'innovation. 
Les innovations radicales sont toutefois rares, d'autant plus au regard des caractéristiques du système de transport. Celui-ci étant par ailleurs non seulement un système technique (avec ses véhicules, ses infrastructures) mais aussi un système social, le déploiement des innovations radicales suppose de faire évoluer les relations entre les acteurs, malgré les inerties inhérentes à des relations inscrites dans le temps long. A cet égard, le rôle de l'Etat doit lui-même être questionné, à l'interface entre acteurs économiques et acteurs de la recherche, car il est un élément essentiel de l'évolution du système social, notamment pour le cas de systèmes de transport au caractère hybride. Nous détaillerons successivement chacun de ces éléments.

\subsection{Les spécificités de l'innovation radicale en transport...et en transport de marchandises}

Trois caractéristiques remarquables peuvent être identifiées :

D'une part, les transports adoptent davantage de technologies qu'ils n'en créent. " Ce qui constitue la spécificité de l'innovation dans le domaine des transports, c'est d'abord la capacité à maîtriser la conception, l'assemblage ou "l'ingénierie » de systèmes complexes - les « objets » comme les avions, les automobiles ou les systèmes de gestion de trafic étant, en effet, parmi les plus complexes qui puissent être utilisés. En conséquence, les logiques de coût et d'organisation des processus de production y jouent un rôle majeur » (Theys, 2005).

D'autre part, la maturité des technologies mobilisées est élevée, et toute évolution n'est dès lors que très souvent marginale.

Par ailleurs, le domaine des transports est à l'interface entre public et privé; les services de transport, et notamment de transport de marchandises, sont largement soumis à concurrence. Toutefois, en tant qu'industrie de réseaux aux caractéristiques spécifiques, rendements croissants, présence d'externalités..., la nécessité d'une régulation publique est forte, voire parfois d'une gestion des activités sous forme de monopole public. Les conséquences pour l'innovation de ce caractère hybride des systèmes de transport sont les suivantes :

-Les services ne peuvent s'envisager indépendamment des infrastructures, financées majoritairement par les acteurs publics et dont la durée de vie est longue; en conséquence une innovation de rupture dans les services peut rencontrer des obstacles à son déploiement si elle suppose de nouvelles infrastructures.

- Le système «social» du transport est corrélativement complexe car il associe une multitude d'acteurs, à la fois publics et privés, constructeurs, opérateurs, usagers, puissance publique ;

- «Les mécanismes de marché sont enfin forcément influencés par la réglementation et les financements publics » (Theys, 2005).

De plus, dans le fret ferroviaire, les marges faibles rendent impossible toute synergie pour dégager de la marge et apporter des innovations au système. Et celui qui fait de la marge ne souhaite pas la voir diminuer et donc s'oppose dans un jeu compliqué d'acteurs à toute évolution/innovation importante.

Le jeu d'acteurs est lui-même complexe: le tractionnaire ferroviaire n'est pas le commissionnaire de transport, il ne possède pas les wagons qui sont la propriété de sociétés différentes de celles qui les exploitent.

Cette difficulté à innover peut donc s'expliquer par une conjonction de raisons : le coût de nouvelles infrastructures éventuelles, la nécessité de coopérations européennes (puisque le créneau privilégié du fret ferroviaire devrait être les grandes distances), et le poids des lobbies routiers et autoroutiers.

\subsection{Des innovations qui s'inscrivent dans un système social}

L'émergence des innovations radicales repose en premier lieu sur des connaissances scientifiques et des compétences techniques. Le manuel d'Oslo de l'OCDE pour l'innovation précise en effet qu'elles constituent un point d'appui essentiel des innovations radicales. Ces connaissances sont souvent 
détenues par les acteurs de la recherche publique, dont la mission est de les produire et de les enrichir. La mise en place des interfaces pertinentes entre la science et l'industrie constitue donc une condition préalable à l'émergence des innovations radicales. En matière de transport, il s'agit de plus de favoriser la rencontre du transport avec d'autres domaines scientifiques, dans la mesure où les évolutions du système de transport reposent pour une large part sur l'intégration de technologies extérieures.

Mais les innovations radicales doivent pour se diffuser modifier l'organisation du système. En effet, elles ne s'adressent pas nécessairement à une demande bien identifiée et quantifiable, ce qui génère des problèmes d'aversion au risque (Perret, 2008), mais elles doivent créer une demande précédemment non exprimée par le marché. Cette demande implique souvent une nouvelle structure du marché et même, l'émergence de nouvelles industries et de nouveaux concurrents. C'est la raison pour laquelle, à la suite de Schumpeter, on parle de "destruction créatrice», de nouveaux acteurs, une nouvelle organisation venant remplacer les anciens. Elles s'inscrivent donc dans un système social, stabilisé par un ensemble de relations entre les acteurs qui marquent la « dépendance du sentier ». La diffusion des innovations radicales nécessite donc d'accompagner l'évolution dans l'organisation du système, c'està-dire dans les relations entre les industriels, les acteurs publics, mais aussi les opérateurs et les utilisateurs. "L'innovation de rupture est dès lors davantage envisagée comme une innovation sociale, que comme l'intégration en amont de technologies nouvelles (piles à combustible, nanotechnologie...)»(Theys, 2005).

Les innovations radicales modifient ainsi la trajectoire technologique des acteurs et les routines organisationnelles du système mais elles vont ainsi modifier les relations et les structures existantes (Di Maggio et Powell, 1983). Or, c'est la structure existante entre des organisations et leurs relations qui contraignent souvent les acteurs dans l'appropriation des opportunités technologiques nouvelles (Lundvall, 1988; Christensen, 1997).

C'est donc le fonctionnement du système de transport qu'il faut ici analyser, les coordinations sur lesquelles il repose et qui peuvent constituer des rigidités au changement. Le fonctionnement du système de transport mérite par ailleurs une attention particulière dans la mesure où il existe une importante concurrence inter mais aussi intramodale. Cet intérêt pour les travaux de sociologie de l'innovation rejoint l'analyse de Jacques Theys qui insiste sur l'importance que peuvent jouer les processus cognitifs - par exemple à travers les «plates-formes technologiques » ou les « foresights »: effet de mobilisation et de stabilisation des stratégies d'acteurs autour de référentiels communs.

Rejoignant ainsi Michel Frybourg (2002), «l'innovation de rupture ne se réduit pas nécessairement à un changement radical de technologie en amont (pile à combustible, nanotechnologie, supraconductivité...) mais peut se situer à l'aval, au niveau des performances, et résulter de l'effet de composition d'une accumulation d'innovations incrémentales ». L'innovation de rupture apparaît dès lors beaucoup plus comme une innovation sociale, un changement de mode d'organisation ou de mode d'usage des techniques que comme l'émergence d'une technologie radicalement nouvelle.

\subsection{Un renouvellement du rôle de l'Etat dans le processus d'innovation et la structuration du système social du transport?}

L'innovation technologique était, jusqu'au milieu du siècle précédent, envisagée comme le fruit d'échanges bilatéraux entre les universités et l'industrie. Le modèle de la Triple Hélice (Etzkowitz et Leydesdorff, 2000) prône un élargissement de ces échanges afin d'intégrer l'ensemble des acteurs de la recherche et de l'innovation, y compris privés, mais aussi les acteurs en charge du transfert de technologies. C'est à cette condition d' "hybridation» des échanges que le modèle associe la circulation facilitée des connaissances vers l'industrie. Cette hybridation s'obtient si et seulement si une interface pertinente organise ces échanges. Il s'agit alors d'une ré-articulation entre les agences publiques, les institutions de recherche et les entreprises et d'une évolution des tâches dévolues à chacun. Les théoriciens de la Triple Hélice basent leurs recommandations sur l'histoire d'innovations radicales récentes comme l'informatique ou les biotechnologies. C'est donc bien le rôle de l'Etat, en charge de mettre en œuvre cette interface pertinente, qui est ici questionné. Le dialogue science- 
industrie-Etat doit ainsi être organisé via des interfaces institutionnelles dont le rôle est l'alignement des objectifs et des schémas de pensée.

Cette question des interfaces et des supports institutionnels est essentielle en matière de transport, mais leur mise en oeuvre rencontre un certain nombre de problèmes pour trois raisons :

- la très grande complexité des systèmes de décision, avec une multiplicité d'acteurs publics et privés - constructeurs, opérateurs, concepteurs, usagers... - intervenant à toutes les échelles ;

- la complexité et la forte inertie des systèmes techniques et organisationnels, qui génèrent des enjeux de coûts et d'organisation du processus de production ;

-la nécessité de mobiliser des connaissances extérieures au domaine des transports.

\subsection{Vers une analyse systémique de l'innovation}

Lundvall met en évidence au travers de sa définition d'un système national d'innovation que l'ensemble des éléments nécessaires à la production, la diffusion et l'utilisation de connaissances nouvelles sont spécifiques à chaque Etat et ancrées dans une culture nationale.

Les facteurs qui favorisent l'éclosion de l'innovation dans les entreprises participent du système national d'innovation. L'OCDE (1998) propose une grille d'analyse des composantes d'un système national d'innovation.

Dans une synthèse de la littérature qui fait autorité et livre le cadre conceptuel pour de nombreux travaux empiriques, l'OCDE (1997) propose dans son manuel d'OSLO quatre groupes de facteurs qui influencent le comportement innovateur des entreprises : les conditions cadre, la base scientifique et technologique, les facteurs de transfert et la dynamo de l'innovation.

- les conditions cadre désignent les institutions et structures qui définissent le contexte de l'innovation et les règles de leur émergence. Elles comprennent à la fois le système éducatif mais aussi le système financier et réglementaire ;

- la base scientifique et technologique décrit les connaissances initiales et les systèmes de formation et de recherche qui participent de leur diffusion ;

- les facteurs de transfert sont les éléments qui régissent et facilitent les relations entre les acteurs (niveau des liaisons interentreprises, plus ou moins facilité de mobilité, d'essaimage...) ;

- la dynamo de l'innovation renvoie à la capacité d'innovation de l'entreprise, en lien avec son positionnement stratégique comme les compétences de son personnel.

Ce cadre d'analyse renvoie à des déterminants du processus d'innovation à différentes échelles : micro-économique, méso-économique et macro-économique. Ce sont ces différents déterminants que nous tenterons de mettre en évidence au travers de l'analyse d'une variété de projets en France et en Allemagne.

\section{Méthodologie}

\subsection{Les projets sélectionnés}

Les projets suivants ont été sélectionnés en fonction du caractère radical qu'ils revendiquent. 


\begin{tabular}{lll}
\hline & Technology & Description \\
\hline emergents & CaroCap (2002) & In-ground tube for freight transport \\
& Megaswing (2012) & Rail wagon takes semi-trailer on rail \\
& CargoBeamer (2007) & Rail wagon takes semi-trailer pickaback \\
& CargoSpeed (2006) & Bogie to load trailer on rail wagon \\
& CargoMover (2002) & Automated driverless individual rail freight system \\
& CACC (2008) & Connected automated driving of trucks in a platoon \\
\hline successful & SkySails (2001) & Wind propulsion systems for modern cargo vessels \\
& Truck Tolling System (2005) & GPS-based truck tolling on Motorways in Germany \\
& Online Freight Exchange platform (1985) & Demand-Offer-Platform for General Cargo \\
\hline non-successful & CargoLifter (1996) & Airship for heavy goods transport \\
& CargoRapid (2009) & Magnetic, contactless (Transrapid) rail goods transport \\
& RFID (1990 & Radio frequency based coding and information exchange \\
\hline
\end{tabular}

Tableau 1. Les projets allemands revendiquant un caractère d'innovation radicale

\begin{tabular}{lll} 
& Technology & Description \\
\hline emergents & VAM (2008) & Automated goods verhicle \\
& MAREL (2009) & Commodities on electronic infrastructure \\
& R-Shift-Я (2008) & Pickaback system for semi-trailer on rail \\
& DIRISOFT (2007) & Airship for heavy goods transportation \\
\hline successful & MODAHLOR (2006) & Bogie to load trailer on rail wagon \\
& & \\
\hline non-successful & Pickaback Wagons AFR (-) & Versatile pickaback rail wagon \\
& COMMUTOR (1994) & Automated management system at trimodal ports
\end{tabular}

Tableau 2. Les projets français revendiquant un caractère d'innovation radicale

Il faut noter le poids important des projets ferroviaires dans notre échantillon. Eu égard à la domination du mode routier, les projets portant sur le mode ferroviaire supposent inévitablement une évolution radicale des organisations de transport actuelles. Toutefois, ce biais ferroviaire n'est pas neutre, en raison des spécificités du jeu d'acteurs (gestionnaire d'infrastructures, opérateurs historiques, nouveaux entrants...) et des coûts d'infrastructures.

Parmi cette sélection, les projets suivants ont fait l'objet d'un entretien :

\section{Projets allemands sélectionnés}

Cargo Cap : le métro automatique de fret comme réponse à la congestion routière

Cargo Speed : système de ferroutage reposant sur l'utilisation d'un wagon surbaissé

Cargo Rapid : le train de fret à sustentation magnétique: vitesse et espoir de rendement énergétique

Cargo Beamer : le transbordement horizontal pour le transport combiné

CACC : la route automatique pour le fret pour améliorer la sécurité routière

Skysails : cerf-volant comme assistance de traction.

\section{Projets français sélectionnés}

VAM : les wagons automoteurs comme réponse au problème du wagon isolé

Modalohr : le développement de l'autoroute ferroviaire

Commutor : le transbordement rapide de charges lourdes et conteneurs

R Shift R : logistique optimisée du transport de fret pour permettre un report modal

Dirisoft : le dirigeable pour les charges lourdes 
Les projets allemands sont des projets récents (2001 à 2009), alors que les projets français sont parfois plus anciens : Commutor (85), R-Shift-R (08), Modalohr (03), VAM (09) et le réseau de recherche Dirisoft (07). Tous les projets sélectionnés revendiquaient une forme de radicalité.

\subsection{La grille d'entretien}

Les entretiens menés s'adressaient à différents représentants : porteurs de projet, directeurs d'entreprise, inventeurs.

Le même schéma d'analyse a été conservé en France et en Allemagne, avec trois échelles d'analyse :

- une échelle macro qui porte sur les institutions et le contexte économique général ;

- une échelle méso sur la structure du système d'acteurs et son évolution ;

- une échelle micro sur l'organisation et la structuration du projet.

\section{L'analyse des processus d'innovation en France et en Allemagne: les résultats de nos entretiens}

Les résultats de nos entretiens montrent l'importance conjointe des déterminants aux trois niveaux $\mathrm{micro} / \mathrm{méso} / \mathrm{macro}$.

A l'échelle micro du projet, la bonne coordination et les partenariats sont essentiels ;

à l'échelle méso de la «branche» ou du système de transport au-delà du seul projet, les jeux d'acteurs et positionnement des acteurs dominants, notamment pour le mode ferroviaire, sont des éléments déterminants du succès ou de l'échec d'un projet. Une implication de l'ensemble du système d'acteurs est donc à construire.

À l'échelle macro, on notera le rôle du soutien politique et du lobbying.

\subsection{Les déterminants micro-économiques de la trajectoire du processus d'innovation}

Dans les projets interrogés, on retrouve une organisation analogue en France et en Allemagne :

- Une entreprise privée en étroite relation avec l'inventeur, une direction de la recherche d'entreprise privée, un réseau de recherche

- Des partenaires R\&D privés ou publics

-Des industriels

L'inventeur : une personnalité déterminante, d'animation et de coordination.

La dimension humaine apparait cruciale dans le discours des personnes interrogées. La personnalité du porteur de l'innovation est déterminante. D'abord parce le réseau de l'individu et sa capacité à savoir vendre son projet sont importants, mais aussi en ce qui concerne sa capacité à mobiliser les équipes. En effet, un des éléments de réussite d'un projet passe par la bonne coordination entre les équipes. La coordination est essentielle pour aboutir à une vision commune, d'autant plus sur les technologies véritablement innovantes.

Or, si les inventeurs sont d'incontestables spécialistes techniques, l'aboutissement du projet nécessite également des compétences de management de projet spécifiques qui font souvent défaut, sans structures d'interface.

A cet égard, la mise en place de structures juridiques pour poursuivre le développement de l'innovation se retrouve dans presque tous les projets allemands, contre très peu en France. Par ailleurs, ces structures sont mises en place très tôt, alors qu'elles marquent véritablement la mise en marché de l'innovation en France. 
Il s'agit parfois d'un réseau de sociétés pour promouvoir un système clé en main. Ainsi, on peut citer CargoBeamer AG et ses filiales CargoBeamer Rolling Stock GmbH, CargoBeamer intermodal operations $\mathrm{GmbH}$ et CargoBeamer Terminal GmbH. Le premier wagon produit en série (CargoBeamer JetModule Sdkmss) a été livré en avril 2013.

CargoBeamer intermodal operations $\mathrm{GmbH}$ s'occupe des clients de CargoBeamer. L'entreprise travaille avec les wagons, les terminaux et le portail de réservation eLogistics de CargoBeamer. Rolling Stock se charge des wagons et de leur entretien. CargoBeamer AG accompagne, avec sa filiale « Terminal », les entreprises qui gèrent des terminaux et leurs gérants pour la livraison d'installations, la mise en service, l'exploitation et l'entretien des installations de transbordement. Un système de gestion de terminal est installé pour la gestion de celui-ci.

Toutefois, dans les deux pays, il demeure des lacunes dans la prise en compte de la dimension économique des projets. Le besoin d'éclairage complémentaire a priori parmi les plus attendus d'une démarche d'innovation, tel qu'il ressort de plusieurs entretiens, est l'approche économique. L'innovation non technologique, et notamment sur les renversements de modèles économiques, deviendra pourtant aussi critique que l'innovation technologique dans la conception qu'en ont des managers.

Des partenariats essentiels dans un système de transport qui est aussi un système social.

Si les partenariats industriels sont nécessaires au financement des projets, l'implication de ces industriels est aussi essentielle pour positionner le projet dans une logique de marché.

Dès l'origine et les essais, Cargo Beamer s'est appuyé sur :

- les terminaux conventionnels de Deutschen Umschlaggesellschaft Schiene Straße, DUSS (société allemande de transbordement rail-route) appartenant aux réseaux DB

- Transa Spedition, également une filiale de la DB qui a volontiers mis ses semi-remorques à disposition

Ainsi, depuis juin 2013, CargoBeamer, en coopération avec Volkswagen AG, transporte des marchandises sur le trajet Wolfsburg - Barcelone et construit à cet effet une installation de transbordement dans l'usine Volkswagen de Wolfsburg.

La mise en place de l'autoroute ferroviaire en France résulte dans la même logique de la signature en 2005 d'un protocole d'accord formalisant l'engagement des parties de coopérer dans l'objectif d'une mise en service au 31 mars 2007. Les signataires étaient le ministre des transports français, la société Autoroutes du Sud de la France, la Caisse des dépôts et consignations, SNCF-Participations, Réseau ferré de France, la société Modalohr, la SNCF, l'organisation professionnelle «Transport et Logistique de France », et l'Agence de financement des infrastructures de transport de France.

On peut constater que les Allemands mobilisent des PME davantage que les gros acteurs. En Allemagne il y a en effet un Mittelstand bien établi (entreprises de plus ou moins 60 salariés, avec une participation des salariés à une gouvernance collective) qui a de bonnes performances d'innovation.

Par ailleurs, la structuration syndicale des différentes filières est plus forte, ce qui facilite l'accès à un ensemble d'acteurs et leur mobilisation collective.

Le système d'innovation en France est au contraire plus petit, articulé autour de l'inventeur et des partenaires industriels. Les partenaires en France sont des acteurs dominants représentatifs du système établi. Cet élément est notamment problématique en matière ferroviaire, où de nombreux projets, en France comme en Allemagne, rencontrent la réticence de l'opérateur historique.

Démarche bottom-up vs démarche top down: une implication différente des industriels. 
En Allemagne, les industriels financent dès le départ et ont une démarche commerciale proactive. L'approche bottom up est privilégiée en Allemagne avec des réseaux financés en grande partie par les cotisations des membres et par les contrats obtenus avec les industriels.

Dans la conception d'un projet en France, les porteurs cherchent également le soutien des industriels. Toutefois, la présence de ces derniers ne signifie pas qu'ils vont s'engager ensuite dans le financement d'un prototypage. Leur présence peut s'expliquer alors par des logiques de manifestation d'intérêts. Les industriels se positionnent, essayent de rester en contact avec les technologies nouvelles, en attendant de connaitre la position de l'Etat, c'est-à-dire de savoir s'il est intéressé ou non pour soutenir le projet (c'est le cas de $3 / 5$ des projets).

\subsection{L'échelle méso : la nécessaire prise en compte de l'ensemble des acteurs de la supply chain et de la structure sociale actuelle du secteur}

Un problème de diagnostic amenant à sous-estimer la nécessité d'innovations organisationnelles complémentaires.

Ce sont en effet des compétences essentiellement techniques qui sont mobilisées dans le cadre des projets. Les acteurs mettent en avant le côté radical ; ils insistent sur la rupture technologique, et elle seule. Toute la rupture (organisationnelle) complémentaire qui est nécessaire sur l'organisation du système d'acteurs est totalement ignorée.

Ce problème est exacerbé pour les problèmes ferroviaires où on se heurte à un problème de diagnostic: les difficultés du fret ferroviaire ont longtemps été analysées comme émanant d'inadaptations des techniques d'organisation de la production. Il n'y avait donc pas de négation de l'existence d'un problème chez Fret SNCF, mais il ne méritait pas à proprement parler une intervention des pouvoirs publics par exemple.

Plus précisément, l'intervention des pouvoirs publics était restreinte à l'octroi de financements pour les prototypes notamment, mais les ingénieurs de la SNCF demeuraient les principaux producteurs des diagnostics et des solutions sur les difficultés du fret.

La nécessaire implication de tous les acteurs de la chaîne et notamment des opérateurs, malgré les risques de déstructuration du secteur.

L'analyse des projets met en lumière l'insuffisance association des opérateurs en amont, notamment sur les projets ferroviaires. Commutor qui a semble-t-il été abandonné suite à un problème de wagon, et à certains problèmes de concurrence entre les filiales de la SNCF, ce qui s'est traduit par des pressions de part et d'autre. Il en ressort que l'intelligence des opérateurs doit être prise en compte. On ne peut introduire l'innovation qu'avec l'ensemble des acteurs de la supply chain ; «On ne fait de bonnes innovations que si l'on a avec soi tous les gens qui vont mettre le système en œuvre ».

Une difficulté de mobilisation liée aux risques de déstructuration du secteur.

Le bouleversement de la structure du secteur du transport de marchandises se situe au niveau des innovations radicales, soit du produit, soit du processus, c'est-à-dire à un niveau où l'innovation partielle ou l'amélioration ne sont plus suffisantes pour comprendre toutes les variables sur lesquelles l'entreprise doit redéfinir son positionnement sur le marché. La logistique et l'express ont été les terrains sur lesquels le produit transport est devenu un autre produit, qui a besoin d'un processus de production complètement différent et qui a donné lieu à la naissance d'entreprises jamais apparues auparavant dans l'univers du transport de marchandises et qui peuvent difficilement être inscrites dans le cadre de simple segment d'un secteur, comme s'il s'agissait de simples spécialisations nouvelles.

Les barrières à l'entrée de nouveaux acteurs dans ces territoires de l'innovation radicale sont devenues très élevées, à cause soit du changement de philosophie, qui rend difficile la requalification de l'ancien savoir-faire, soit à l'amplitude des moyens financiers nécessaires à la création de nouvelles entreprises (CEMT, 1999). 
Là encore, la structure des systèmes ferroviaires, articulés autour d'acteurs historiques dominants, renforce les résistances.

La nécessaire prise en compte de l'ensemble du système, incluant les infrastructures.

Les spécificités des systèmes de transport tiennent en effet au coût des infrastructures de transport. Si le projet technologique implique une remise en cause des infrastructures existantes, le projet risque d'être difficile à mettre en œuvre.

«il est très intelligent ce wagon, le seul problème c'est qu'il nécessite actuellement de faire des terminaux très spécialisés. Donc si vous voulez, c'est cela qui manque. Il manque cette logique de pouvoir faire quelque chose qui utilise ce qui existe dans le marché. Il y a des wagons poche, donc il fallait regarder l'infrastructure... »

Par ailleurs là encore, des spécifiques nationales entrent en ligne de compte. Ainsi, les projets de transbordement en faveur du transport combiné sont favorisés en Allemagne par les caractéristiques du réseau. En effet, la lenteur du triage y est d'autant plus problématique que le réseau est très éclaté.

Les spécificités des systèmes de transport ferroviaire : la complexité du jeu d'acteurs.

Le système ferroviaire mobilise en effet une nébuleuse d'acteurs, gestionnaire d'infrastructures, opérateurs ferroviaires, par ailleurs en fréquente évolution, mais également loueurs de locomotives, de wagons, ou encore collectivités territoriales. Ces verrous organisationnels compliquent encore la mobilisation de partenaires, notamment pour le déploiement de projets à l'étranger.

\subsection{L'échelle macro : soutien politique et financier : des enjeux liés}

La difficile quête des moyens financiers.

Il faut à cet égard distinguer les moyens financiers liés au développement des concepts, qui relèvent d'appels d'offre classiques, des moyens financiers nécessaires pour les expérimentations, qui demandent en matière de transport des moyens financiers beaucoup plus importants. Ce dernier point est là encore exacerbé pour les projets ferroviaires. Dès lors, les appels à projets émanant d'institutions publiques ne suffisent plus, et l'aboutissement des projets nécessite de trouver d'autres circuits de financement, notamment du côté des industriels.

Le financement public semble indispensable pour développer l'idée, dans la phase qui suit l'invention à proprement parler. La raison évoquée est la possibilité de développer cette idée dans un environnement protégé, focalisé uniquement sur la faisabilité technique et non directement la valorisation sur les marchés. Le financement public permet aussi de réaliser un prototype ou un démonstrateur. Parfois néanmoins, des critiques ont été formulées, tenant à la lenteur des prises de décision (notamment si des emplois sont en cause), et les difficultés de candidature aux appels à projet comparées aux résultats attendus. De plus, il arrive que les règles de financement ne soient pas compatibles avec l'état du processus d'innovation (par exemple, disposer d'un brevet fait une différence, mais un brevet signifie déjà un stade avancé du projet). Néanmoins, en général, un financement public semble être le cadre pertinent de développement de l'innovation.

En France, le financement public était indispensable au début du projet, mais il s'arrête le plus souvent à la phase d'étude et il est très difficile d'obtenir des financements pour aller au-delà jusqu'à la phase de prototype.

Le manque de visibilité des circuits.

Si les circuits de financement existent, il donne le sentiment aux acteurs interrogés d'une grande dispersion, nécessitant là encore un accompagnement. 
L'accompagnement des projets va toutefois au-delà du simple montage technique de dossiers. C'est ici d'une véritable interface qu'il s'agit, capable de valoriser les aspects techniques du projet à bon escient et de proposer les complémentarités pertinentes.

\section{Une volonté politique forte.}

Sans un soutien fort de l'Etat, rien n'est possible. Ce constat est partagé par l'ensemble des interlocuteurs comme étant l'obstacle majeur de l'innovation.

Toutefois, la question du rôle de l'Etat est ici envisagée uniquement en termes de financement. On constate qu'en termes de comparaison franco-allemande, la France est plutôt bien placée en termes de moyens mis à la disposition de la recherche publique, mais pas en termes de résultats; il y a donc un problème d'efficacité dans l'allocation des moyens. L'Allemagne semble un peu moins dans la question du financement et plus dans celle des services : de marketing, d'aide, mais peut-être pas de financement direct. Sur les 5 projets français interrogés, on peut aussi constater que les deux projets qui ont bénéficié d'un soutien de l'Etat sont allés jusqu'à la phase de déploiement d'un démonstrateur.

La question est toutefois de savoir si le rôle de l'Etat doit se limiter à des financements. Il semble plutôt qu'il s'agit de porter sur la sphère politique les problèmes du fret de façon homogène. Ainsi concernant COMMUTOR, les voies étaient discordantes. Pour N. Neiertz, l'administration de l'Equipement, au début des années 1990, connaissait un affrontement entre ingénieurs des Ponts, entre ceux qui soutenaient le mode routier et ceux qui soutenaient le mode ferroviaire. Selon l'auteur, cet affrontement peut s'expliquer par la période de choix fondamentaux en matière d'infrastructures que la France traverse depuis la signature du Traité de Maastricht qui aborde la question des réseaux transnationaux, et par la menace que constituerait pour les routiers un essor rapide des transports combinés (Lemettre, 2005). On ne peut ainsi pas dire qu'il y ait eu une négation des difficultés du fret ferroviaire, mais la situation ne paraissait pas clairement formulée en problème public, autour duquel des groupes identifiés se mobiliseraient pour influencer sa définition et l'inscrire à l'agenda institutionnel. Malgré la variété des définitions du «problème public », la plupart insistent sur deux dimensions : le fait qu'une situation doive être transformée en problème, le fait que ce problème soit considéré dans la sphère publique et devienne un objet légitime de l'action publique. Or, ces deux dimensions ont posé question pour le fret ferroviaire. Jusqu'au début des années 1990 en France, le problème du fret ferroviaire demeurait discret et relativement confiné dans la SNCF et un cercle restreint de spécialistes du secteur des transports. Au début des années 1990, c'est principalement l'avenir du mode ferroviaire en général qui va être discuté dans l'arène politique. Avec l'arrivée de J.C. Gayssot à la tête du Ministère de l'Equipement va s'ouvrir clairement une nouvelle ère de la politique française des transports avec l'inscription claire du problème du fret ferroviaire à l'agenda gouvernemental.

Par ailleurs, en Allemagne, le soutien est davantage politique que financier. Concernant le projet CargoCap, le ministre des Transports de la Rhénanie du Nord-Westphalie, Lutz Lienenkämper, pense que le projet est "une alternative à prendre au sérieux" dans le secteur des transports. Il n'y aura cependant pas d'argent du Land pour la réalisation du projet.

\section{Un rôle ambigu des pôles de compétitivité.}

En Allemagne, pour deux projets étudiés, les responsables des clusters n'ont été d'aucun support. Ces responsables de clusters comme de lobbies sont alors vus comme des représentants des systèmes établis et des acteurs dominants.

En France, pour 3/5 des projets, être labellisé, c'était uniquement pour avoir l'opportunité d'un financement conséquent derrière. 


\section{Conclusion}

L'analyse de quelques projets d'innovations radicales en France et en Allemagne, et notamment des conditions de leur émergence confirme la nécessité, souvent éludée, de penser l'innovation sociale au sein du système d'acteurs, conjointement à l'innovation technologique qui constitue souvent le cœur du projet.

A cet égard, les modalités des partenariats sont très différentes entre la France et l'Allemagne, 1'Allemagne associant davantage de PME que des acteurs établis et dominants comme c'est le cas en France. Toutefois, les partenariats à établir doivent non seulement concerner les industriels, mais aussi les opérateurs et les gestionnaires d'infrastructures. C'est bien l'ensemble des acteurs du système de transport qu'il convient de mobiliser, ce qui est rarement fait. Or, l'inertie ou les difficultés de déploiement proviennent le plus souvent des risques de déstructuration du secteur, alors même que l'inventeur s'est concentré pour sa part sur les dimensions techniques du projet, en éludant les dimensions économiques et organisationnelles.

Enfin, la nécessité du dialogue science-industrie-Etat y est réaffirmée. On constate toutefois le manque ou la relative inefficacité des structures d'interface existantes. L'évolution du rôle de l'Etat dans la dynamique d'innovation du système de transport est donc essentielle. Il est pour cela primordial de clarifier les buts ultimes assignés aux politiques de valorisation.

Enfin, l'innovation radicale qui affecte aujourd'hui le secteur des transports de marchandises pose des problèmes d'identification du secteur même. Peut-on parler encore d'un secteur transport de marchandises ? N'y a-t-il pas plutôt un mégasecteur gestion des flux ? Dans la complexité des opérations réalisées à l'intérieur de ce mégasecteur, le transport ne sera-t-il pas de plus en plus conçu comme traction, comme maillon pauvre de la chaîne ? Et qui sera disposé à faire des investissements dans le maillon pauvre? Le risque que le secteur des transports terrestres devienne un segment traction, et que le segment traction devienne le moins rentable, le moins qualifié, le moins séduisant est un risque réel (CEMT, 1995).

\section{Bibliographie}

Bauer R. (2006). Gescheiterte Innovationen: Fehleschläge und technologischer Wandel. Campus Verlag, Auflage 1 (24. April 2006). ISBN-13: 978-3593379739

Benner M. and Tushman M. L. (2003): Exploitation, Exploration and Process Management: The Production Dilemma Revisted. In: Academy of Management Review. 28. Nr. 2. pp. 238-256.

BMBF (2010). Ideen. Innovation. Wachstum. Hightech-Strategie 2020 für Deutschland. Bundesministerium für Bildung und Forschung (editor). Bonn, Berlin 2010

Borgwardt A. (2009). Warum scheitern, wann gelingen Innovationen?. Konferenzbericht der Veranstaltung Forschungsund Innovationspolitik in Deutschland. Friedrich-Ebert-Stiftung. 7. Mai 2009

Chanal V. and Mothe C. (2004) : Quel design organisationnel pour combiner innovation d'exploration et innovation d'exploitation. Actes de la XIIIème Conférence Internationale de Management Stratégique 'A.I.M.S.'

Christensen T. A., Lämmer-Gamp T. and Meier zu Köcker G. (2012), Let's make a Perfect Cluster Policy and Cluster Programme - Smart Recommendations for Policy Makers, Berlin/Copenhagen, 2012

Cooke P., Uranga M.G., Etxebarria G. (1997). Regional innovation systems: Institutional and organizational dimensions. In: Research Policy 26 (1997). pp. 475-491

Dahlin K. B. and Behrens D. M. (2005). When is an invention really radical? Defining and measuring technological radicalness. In: Research Policy 34 (5), pp. 717-737.

Davis C.H., Arthurs D., Cassidy E. and Wolfe D. (2006). What Indicators for Cluster Policies in the 21th Century? In: Proceedings of Blue Sky II 2006, Ottawa, September 2006

European Commission (2011). White Paper. Roadmap to a Single European Transport Area - Towards a competitive and resource efficient transport system. Brussels. 28.3.2011 
European Communities (2008). The Concept of Clusters and Cluster Policies and their Role for Competitiveness and Innovation: Main Statistical Results and Lessons Learned. Commission Stuff Working Document SEC (2008) 2637. Luxembourg 2008

Fagerberg J. and Srholec M. (2008), National innovation systems, capabilities and economic development, Research Policy, Volume 37, Issue 9, October 2008, pp. 1417-1435

Filarski R. (2004): The rise and decline of transports systems. Ministry of transport and public Works. Netherlands. TRC

Freeman C. and Perez C. (1988). Structural crises of adjustment, business cycles and investment behaviour. In: Technical change and economic theory. Dosi G., Freeman C., Nelson R., Silverberg G., Soete L. (editors). London: Pinter. 1988. Chapter 3

Furman J. L., Porter M. E., Stern S. (2002). The determinants of national innovative capacity. In: Research Policy, Volume 31, Issue 6, August 2002, pp. 899-933

Gans J., Stern S. and Intellectual Property Research Institute of Australia (2003). Assessing Australia's innovative capacity in the 21st century. Intellectual Property Research Institute of Australia, Melbourne, Vic

Geels F. W. (2010). Ontologies, socio-technical transitions (to sustainability), and the multi-level perspective. In: Research Policy. Volume 39. Issue 4. May 2010. pp. 495-510

Goldstein J.S. (1988). Long Cycles: Prosperity and War in the Modern Age. Yale University Press: New Haven and London 1988

He Z.-L. and Wong P.-K. (2004): Exploration vs. exploitation: An empirical test of the ambidexterity hypothesis. Organ. Sci. 15. pp. 487-494.

Hellström T. (2003). Systemic innovation and risk: Technology assessment and the challenge of responsible innovation. In: Technology in Society. Volume 25. Issue 3. August 2003. Pp. 369-384

Hu M.C. and Mathews J. A. (2005). National innovative capacity in East Asia. In: Research Policy. Volume 34 . Issue 9. November 2005. pp. 1322-1349

Linde C. v. d. (2002). A cluster template as part of Cluster Meta Study. Harvard Business School. http://data.isc.hbs.edu/cp/index.jsp (last access: 17.06.2013)

Mensch G. (1975). Das technologische Patt: Innovationen überwinden die Depression. Fischer Taschenbuch Verlag. November 1977. (780-ISBN-3-596-21978-7)

Meyer-Stamer J. (2009). Moderne Industriepolitik oder postmoderne Industriepolitiken? Schriftenreihe Moderne Industriepolitik. 1|2009. IBN: 978-3-86872-113-3

Natário M., Couto J., Braga A. M., and Tiago T. M. (2011). Evaluating The Determinants Of National Innovative Capacity Among European Countries. In: ERSA conference papers (No. ersa10p1342). European Regional Science Association.

Norman D. A. and Verganti, R. (2012). Incremental and radical innovation: design research versus technology and meaning change. manuscript submitted to Design Issues. March 2012

O’Reilly C. A. and Tushman M. L. (2004): The ambidextrous organization. Harvard Bus. Rev. 83 pp. 74-81.

OECD (2007). Competitive Regional Clusters - National Policy Approaches. OECD Reviews of Regional Innovation (ISBN 978-92-64-03182-1)

Porter M. E. (1990). The competitive advantage of nations. In: Harvard Business Review. March/ Avril 1990

Porter M. E. and Scott S. (2003). Ranking national innovative capacity: findings from the national innovative capacity index. The Global Competitiveness Report 2004 (2003): 91-115.

Porter M. E. and Stern S. (2001). National innovative capacity. The global competitiveness report, 2002. pp. 102-118.

Sanidas E. (2004). Technology, technical and organizational innovations, economic and societal growth. In: Technology in Society. Volume 26. Issue 1. January 2004. pp. 67-84

Schumpeter J.A. (1944): Capitalism, Socialism and Democracy. Second Impression 1944. George Allen \& Unwin LTD. London.

Suarez- Villa L. (1990). Invention, inventive learning, and innovative capacity. System Research and Behavioral Science. Volume 35, Issue 4, pp. 290-310,

Sundbo J. and Gallouj F. (1998). Innovation as a loosely coupled system in services. Paper presented at the CRIC workshop on Innovation and Services, 17-18 March 1998, Manchester

Swann G. M. P. (2009). The Economcs of Innovation: An Introduction. Edward Elgar Publishing, Cheltenham 2009 
Theys J. (2005). Quelles technologies clefs pour l'Europe? Les enjeux liés au transport. Rapport pour la DG recherche de la Commission Européenne 